\title{
Inverse magnetocaloric effect in polycrystalline $\mathrm{La}_{0.125} \mathrm{Ca}_{0.875} \mathrm{MnO}_{3}$
}

\author{
Anis Biswas $*$ Tapas Samanta $t$ S. Banerjee, and I. Dast \\ Saha Institute of Nuclear Physics, $1 / A F$, \\ Bidhannagar, Kolkata 700 064, India
}

\begin{abstract}
Recently the inverse magnetocaloric effect is observed for different compounds. However there is very rare for any manifestation of the effect to be seen in manganites. We have found inverse magnetocaloric effect in the case of polycrystalline $\mathrm{La}_{0.125} \mathrm{Ca}_{0.875} \mathrm{MnO}_{3}$. Such phenomenon is attributed to the stabilization of antiferromagnetic state associated with inherent magnetic inhomogeneous phases for this compound.
\end{abstract}

PACS numbers: 75.30.Sg, 75.47.Lx

*Electronic address: anis.biswas@saha.ac.in

${ }^{\dagger}$ Electronic address: tapas.samanta@saha.ac.in

${ }^{\ddagger}$ Electronic address: indranil.das@saha.ac.in 
The study of the magnetocaloric effect (MCE) has been a subject of intense research owing to its possible application in magnetic refrigeration [1, 2, 3, 4, 5, 6, 7, 8, 9, 10, 11, 12, 13, 14, 15, 16, 17, 18]. It has been widely observed that the magnetic entropy reduces with the application of magnetic field for different materials including some paramagnetic salts [1]. The cooling can be possible by utilizing those materials subject to expose them in the magnetic field followed by demagnetizing them adiabatically. However, there is recent report on the discovery of materials with an inverse situation in which, the magnetic configuration entropy increases due to the application of the magnetic field [4]. Such an effect is known as inverse magnetocaloric effect (IMCE). For the materials exhibiting IMCE, the attaining of low temperature can be possible by only just magnetizing them adiabatically [4]. Some of the examples of such materials are NiMnSn, FeRh, TbNiAl, DySb, $\mathrm{Tb}_{2} \mathrm{Ni}_{2} \mathrm{Sn}, \mathrm{NiMnSb}$ etc [4, 19, 20, 21, 22, 23, 24, 25]. The materials which show IMCE, can be used as heatsink for heat generated when a conventional magnetocaloric material is magnetized before cooling by demagnetization under adiabatic condition [4, 18]. The refrigeration efficiency can be enhanced by using materials exhibiting IMCE in composites with conventional magnetic refrigerants [4]. Therefore searching of suitable materials, which display IMCE is an important issue in the ongoing research related to the magnetic refrigeration. Our present study is based on the magnetocaloric property of polycrystalline $\mathrm{La}_{0.125} \mathrm{Ca}_{0.875} \mathrm{MnO}_{3}$. We have observed IMCE with quite large value of change of magnetic entropy $(-\Delta S)$ in this compound.

The perovskite manganites with general formula $\mathrm{R}_{1-x} \mathrm{~B}_{x} \mathrm{MnO}_{3}$ ( $\mathrm{R}$ is rare-earth, $\mathrm{B}$ is bivalent ion) are considered potential magnetic refrigerants [2, 13, 14, 15, 16, 17]. $\mathrm{La}_{1-x} \mathrm{Ca}_{x} \mathrm{MnO}_{3}$ is a manganite system, which has a very rich phase diagram depending on the values of $\mathrm{x}$ [26]. Although some works regarding the magnetocaloric properties of this system with $\mathrm{x} \sim 0.2-0.5$ are reported, there is hardly any such study for high doping concentration of bivalent ion [2]. In fact, a little attention has been paid to the study of MCE for other manganite systems in high doping region (i.e, high value of $\mathrm{x}$ ) also. We have chosen $\mathrm{La}_{0.125} \mathrm{Ca}_{0.875} \mathrm{MnO}_{3}$ for two main reasons. Firstly, it is a system with high doping concentration of bivalent ion. Secondly, its position in the phase diagram of $\mathrm{La}_{1-x} \mathrm{Ca}_{x} \mathrm{MnO}_{3}$ is at phase boundary between antiferromagnetic (AFM) and canted antiferromagnetic (CAF) phases [26]. Therefore this compound provides an opportunity to study the effect of the phase boundary on magnetocaloric property of a system as well. There are 
reports of the observation of IMCE in the chrage ordered systems such as $\operatorname{Pr}_{0.5} \mathrm{Sr}_{0.5} \mathrm{MnO}_{3}$, $\mathrm{Nd}_{0.5} \mathrm{Sr}_{0.5} \mathrm{MnO}_{3}$ [27, 28]. For that systems, charge order transition and antiferromagnetic transition occurs simultaneously [27, 28]. In complete contrast to those cases, in the present system charge ordering hardly occurs. In spite of this, the system exhibits IMCE.

The polycrystalline $\mathrm{La}_{0.125} \mathrm{Ca}_{0.875} \mathrm{MnO}_{3}$ was prepared by sol-gel method. The details of the solgel method has been described in our previous article [29]. At the end of the solgel process, the decomposed gel was annealed at $1400^{\circ} \mathrm{C}$ for 36 hours. The x-ray powder diffraction study has confirmed the formation of the sample with single crystallographic phase with PNMA space group. The lattice parameters are determined as, $\mathrm{a}=5.347 \AA \mathrm{A}, \mathrm{b}$ $=7.442 \AA$, and $\mathrm{c}=5.318 \AA$.

A commercial SQUID magnetometer was utilized for magnetization study. The temperature dependence of dc susceptibility (Fig. 1) shows clear antiferromagnetic transition at $\sim 120 \mathrm{~K}$. The transition temperature is consistent with the phase diagram of the sample [26]. The magnetization measurement has been performed in the presence of 100 Oe magnetic field in zero field cooled protocol. We have also done the specific heat study using semi-adiabatic heat pulse method. The transition is manifested by observed maxima in the temperature dependence of specific heat as well (inset of Fig. 1).

The isothermal magnetic field dependence of magnetization $[\mathrm{M}(\mathrm{H})]$ at different temperatures has been studied for the sample (Fig. 2). To get intricate details of $\mathrm{M}(\mathrm{H})$, we have examined Banerjee's plot [30] i.e., H/M vs. $\mathrm{M}^{2}$ behavior around transition temperature (inset, Fig. 2). The negative slope of Banerjee's plot is evident in high magnetic field region (above $\sim 35 \mathrm{kOe}$ ), which is characteristic of first order transition [30]. From the isothermal $\mathrm{M}(\mathrm{H})$ curves, the change of the magnetic entropy $(-\Delta S)$ was estimated for various magnetic fields by using the Maxwell's relation [1],

$$
\left(\frac{\partial S}{\partial H}\right)_{T}=\left(\frac{\partial M}{\partial T}\right)_{H}
$$

The temperature dependence of $-\Delta S$ for different magnetic field has been shown in Fig. 3. A minimum in $-\Delta S(\mathrm{~T})$ has been observed at $\sim 120 \mathrm{~K}$, where antiferromagnetic transition occurs. One important feature of $-\Delta S(\mathrm{~T})$ is that the value of $-\Delta S$ remains negative for all the magnetic field at transition temperature for this sample. The negative value of $-\Delta S$ increases with the increase of magnetic field (i.e., the magnetic configuration entropy increases) and it reaches $\sim-6.4 \mathrm{~J} / \mathrm{kg} \mathrm{K}$ for the magnetic field change $0-70 \mathrm{kOe}$. The 


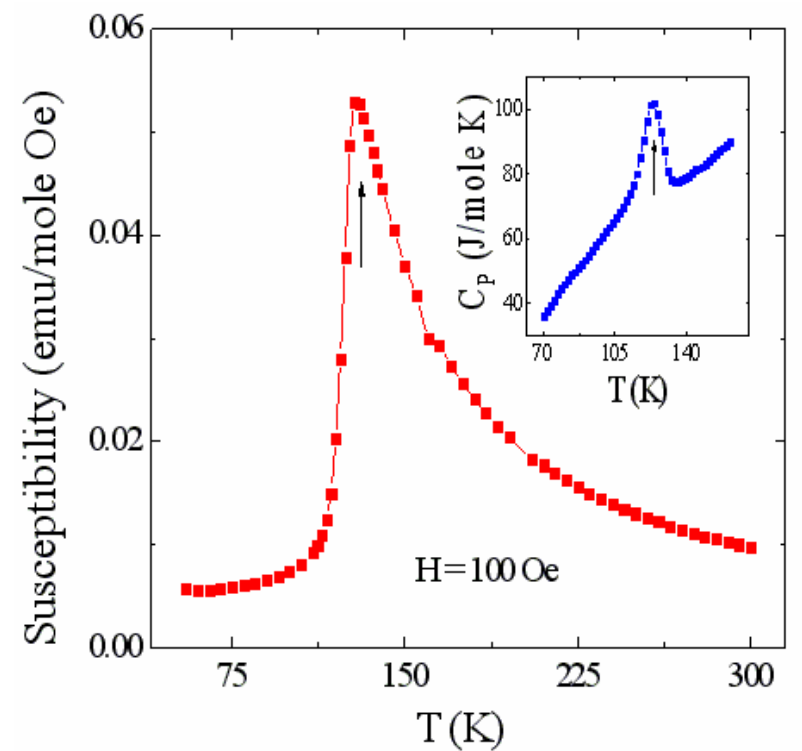

FIG. 1: Temperature dependence of dc susceptibility for polycrystalline $\mathrm{La}_{0.125} \mathrm{Ca}_{0.875} \mathrm{MnO}_{3}$ in the precence of 100 Oe magnetic field. Inset: temperature dependence of specific heat for this sample in the absence of magnetic field. Antiferromagnetic transition is indicated by arrow.

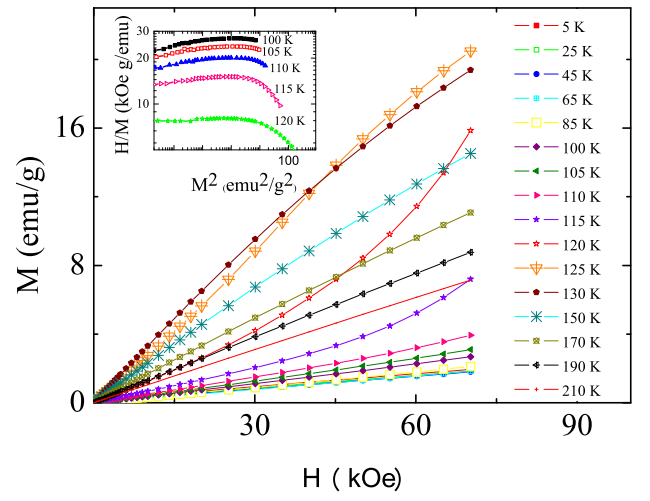

FIG. 2: Magnetic field dependence of magnetization for polycrystalline $\mathrm{La}_{0.125} \mathrm{Ca}_{0.875} \mathrm{MnO}_{3}$ at different temperatures. Inset: $\mathrm{H} / \mathrm{M}$ versus $\mathrm{M}^{2}$ for the same sample at different temperatures around transition temperature.

increase of the negative value of $-\Delta S$ with magnetic field at transition temperature has been shown in inset (b) of Fig. 3. From the magnetocaloric behavior of the sample, it seems that although there is change of slope in Banerjee's plot at high magnetic field, the antiferromagnetism still exists in the field up to $\sim 70 \mathrm{kOe}$. Recently, Ranke et al., put forward a theoretical framework of the magnetocaloric properties of antiferromagnetic system [31]. 


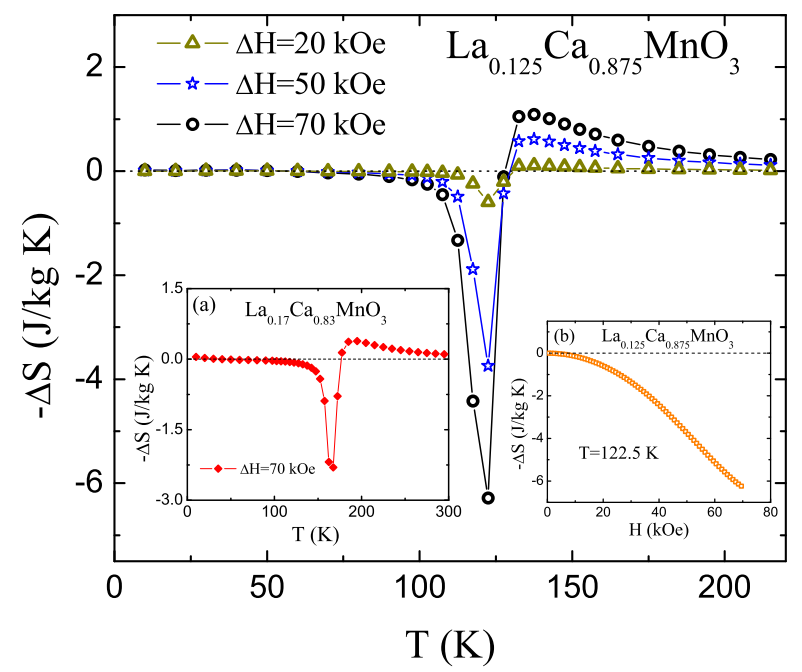

FIG. 3: The temperature dependence of $-\Delta \mathrm{S}$ for $\Delta \mathrm{H}=20,50$, and $70 \mathrm{KOe}$ in the case of $\mathrm{La}_{0.125} \mathrm{Ca}_{0.875} \mathrm{MnO}_{3}$. Large change in magnetic entropy has been observed around transition temperature. Inset (a): $-\Delta \mathrm{S}$ vs. $\mathrm{T}$ for $\Delta \mathrm{H}=70 \mathrm{kOe}$ in the case of $\mathrm{La}_{0.17} \mathrm{Ca}_{0.83} \mathrm{MnO}_{3}$ Inset (b): the magnetic field dependence of $-\Delta \mathrm{S}$ around the transition temperature for $\mathrm{La}_{0.125} \mathrm{Ca}_{0.875} \mathrm{MnO}_{3}$.

The temperature dependence of $-\Delta S$ for $\mathrm{La}_{0.125} \mathrm{Ca}_{0.875} \mathrm{MnO}_{3}$ follow that theoretical model quite convincingly. Previously, we have also observed small negative $-\Delta S$ at antiferromagnetic transition temperature for other manganite system at magnetic field below the required field for quenching the antiferromagnetism as well [14].

Now question arises about the origin of the enhancement of magnetic configuration entropy with the increase of magnetic field. According to the phase diagram (temperature vs $\mathrm{x}$ ) of $\mathrm{La}_{1-x} \mathrm{Ca}_{x} \mathrm{MnO}_{3}, \mathrm{La}_{0.125} \mathrm{Ca}_{0.875} \mathrm{MnO}_{3}$ situates at the phase boundary between antiferromagnetic and inhomogeneous canted antiferromagnetic state (CAF) [26]. It is obvious that CAF phase may have influence on the magnetic state of the sample. As a result of which, magnetically inhomogeneous phase with mixed magnetic exchange interactions can be stabilized at the antiferromagnetic transition temperature. The enhancement of magnetic configuration entropy with the application of magnetic field can occur for such system giving rise to IMCE [4] with large value of $-\Delta S$. We have also studied magnetocaloric property of another $\mathrm{La}_{1-x} \mathrm{Ca}_{x} \mathrm{MnO}_{3}$, with slightly different value of $\mathrm{x}(\mathrm{x} \sim 0.83)$. That sample is of polycrystalline form and prepared in similar condition as $\mathrm{La}_{0.125} \mathrm{Ca}_{0.875} \mathrm{MnO}_{3}$. IMCE is also 
observed for that compound at its antiferromagnetic transition temperature [inset (a), Fig. 3]. However the value of $-\Delta S$ is considerable less for $\mathrm{La}_{0.17} \mathrm{Ca}_{0.83} \mathrm{MnO}_{3}$ in comparison with $\mathrm{La}_{0.125} \mathrm{Ca}_{0.875} \mathrm{MnO}_{3}$. According to the phase diagram of $\mathrm{La}_{1-x} \mathrm{Ca}_{x} \mathrm{MnO}_{3}$, the magnetic transition in the case of $\mathrm{La}_{0.17} \mathrm{Ca}_{0.83} \mathrm{MnO}_{3}$ is paramagnetic to antiferromagnetic, in which there is no influence of CAF phase [26]. From the comparison of the magnetocaloric properties of the two compounds, it can be argued that the presence of magnetically inhomogeneous CAF state plays a vital role in IMCE and the magnetic entropy change becomes significantly enhanced as because of the influence of such state.

To summarize, we have observed IMCE in the case of polycrystalline $\mathrm{La}_{0.125} \mathrm{Ca}_{0.875} \mathrm{MnO}_{3}$ with large value of $-\Delta S$ at antiferromagnetic transition temperature. Possibly, the stabilization of inhomogeneous magnetic state for this compound at its antiferromagnetic transition temperature causes the increase in magnetic entropy in the presence of magnetic field. The observation of the enhancement of magnetic configuration entropy with the increase of magnetic field is very rare especially for manganite systems. 
[1] K. A. Gschneidner, Jr., V. K. Pecharsky, and A. O. Tsokol, Rep. Prog. Phys., 68, 1479 (2005).

[2] M. H. Phan, S. Yu, J. Magn. Magn. Mat., 308, 325 (2007)

[3] V. K. Pecharsky, K. A. Gschneidner, Jr., Phys. Rev. Lett., 78, 4494 (1997)

[4] T. Krenke, E. Duman, M. Acet, E. Wassermann, X. Moya, L. Manosa, and A. Planes, Nature materials, 4, 450 (2005).

[5] Tapas Samanta, I. Das, and S. Banerjee, Appl. Phys. Lett., 91, 082511 (2007)

[6] Tapas Samanta, I. Das, S. Banerjee, Appl. Phys. Lett., 91, 152506 (2007)

[7] Z. B. Guo, Y. W. Du, J. S. Zhu, H. Huang, W. P. Ding, and D. Feng, Phys. Rev. Lett., 78, $1142(1997)$

[8] Tapas Samanta, I. Das, S. Banerjee, J. Appl. Phys., 104, 123901 (2008)

[9] M. Phan, S. C. Yu, N. Hur, Appl. Phy. Lett., 86, 072504 (2005)

[10] Tapas Samanta, I. Das, S. Banerjee, J. Phys.:Cond.Mat., 21, 026010 (2009)

[11] Y. Sun, M. Salamon, S. Chun, J. Appl. Phys., 92, 3235 (2002)

[12] Tapas Samanta, I. Das, Phys. Rev. B, 74, 132405 (2006)

[13] Anis Biswas, Tapas Samanta, S. Banerjee, I. Das, J. Appl. Phys., 103, 013912 (2008)

[14] Anis Biswas, Tapas Samanta, S. Banerjee, I. Das, Appl. Phys. Lett., 92, 012502 (2008)

[15] M. Phan, S. Yu, N. Hur, Y. Yeong, J. Appl. Phys., 96, 1154 (2004)

[16] Anis Biswas, Tapas Samanta, S. Banerjee, I. Das, Appl. Phys. Lett., 92, 212502 (2008)

[17] Anis Biswas, Tapas Samanta, S. Banerjee, I. Das, AIP Conf. Proc., 1003, 109 (2008).

[18] R. J. Joenk, J. Appl. Phys., 34, 1097 (1963).

[19] S. Chatterjee, S. Giri, S. majumdar, S. K. De, J. Phys. D: Appl.Phys., 42, 065001 (2009)

[20] M. P. Annaorazov, S. A. Nikitin, A. L. Tyurin, K. A. Asatryan, A. K. Dovletov, J. Appl. Phys., 79, 1689 (1996).

[21] L. Li, K. Nishimura, W. D. Hutchison, Sol. Stat. Comn., 149, 932 (2009).

[22] L. Li, K. Nishimura, W. D. Hutchison, J. Phys.: Conf. Ser., 150, 042113 (2009).

[23] W. J. Hu, J. Du, B. Li, Q. Zhang, Z. D. Zhang, Appl. Phys. Lett., 92, 192505 (2005).

[24] P. Kumar, N. Singh, K. G. suresh, A. K. Nigam, Arxiv:condmatt/0609335.

[25] W.J. Feng, J. Du, B. Li, W.J. Hu, Z.D. Zhang, X.H. Li, Y.F. Deng, J. Phys. D: Appl. Phys., 42, 125003 (2009). 
[26] Colossal Magnetoresistive Oxides Ed. by Y. Tokura, Gordon and Breach Science Publishers, 2000

[27] P. Chen, Y.W. Du, G. Ni, Eur. Phys. Lett., 52, 589 (2000)

[28] P. Sande, L. Hueso, D. Miguens, J. Rivas, F.Rivadulla, M. A. Lopez-Quintela, Appl. Phys. Lett., 79, 2040 (2001)

[29] Anis Biswas, I. Das, C. Majumdar, J. Appl. Phys., 98, 124310 (2005).

[30] S. K. Banerjee, Phys. Lett., 12, 16 (1964).

[31] P. J. Ranke, N. Oliveira, B. P. Alho, E. Plaza, V. S. Sousa, L. Caron, M. S. Reis, J. Phys.:Cond. Mat. 21, 056004 (2009). 